\title{
Some generalizations of Aczél, Bellman's inequalities and related power sums
}

\author{
Xiaoyan Zhou
}

\section{Correspondence:}

zhouxiaoyan2011@yahoo.com.cn

The School of Science, Zhejiang

University of Science and

Technology, Hangzhou 310023,

China

\section{Abstract}

In this paper, we establish some functional generalizations and refinements of Aczél's inequality and of Bellman's inequality. We also establish several mean value theorems for the related power sums.

Mathematics Subject Classification (2010): 26D15; 26D20; 26D99.

Keywords: generalization, Aczél's inequality, Bellman's inequality, mean value theorem, power sums

\section{Introduction}

Let $n$ be a positive integer, and let $a_{i}, b_{i}(i=1,2, \ldots, n)$ be real numbers such that $a_{1}^{2}-\sum_{i=2}^{n} a_{i}^{2} \geq 0$ or $b_{1}^{2}-\sum_{i=2}^{n} b_{i}^{2} \geq 0$. Then Aczél's inequality [1] can be stated as follows

$$
\left(a_{1}^{2}-\sum_{i=2}^{n} a_{i}^{2}\right)\left(b_{1}^{2}-\sum_{i=2}^{n} b_{i}^{2}\right) \leq\left(a_{1} b_{1}-\sum_{i=2}^{n} a_{i} b_{i}\right)^{2},
$$

with equality if and only if the sequences $a_{i}$ and $b_{i}$ are proportional.

The Aczél inequality (1) plays an important role in the theory of functional equations in non-Euclidean geometry. During the past years, many authors have given considerable attention to this inequality, its generalizations and applications [2-11].

As an example, the Hölder-like generalization of the Aczél inequality (1), derived by Popoviciu [12], takes

$$
\left(a_{1}^{p}-\sum_{i=2}^{n} a_{i}^{p}\right)^{\frac{1}{p}}\left(b_{1}^{q}-\sum_{i=2}^{n} b_{i}^{q}\right)^{\frac{1}{q}} \leq a_{1} b_{1}-\sum_{i=2}^{n} a_{i} b_{i}
$$

where $n$ is a positive integer, and $p, q, a_{i}, b_{i}(i=1,2, \ldots, n)$ are positive numbers such that $p^{-1}+q^{-1}=1, a_{1}^{p}-\sum_{i=2}^{n} a_{i}^{p}>0$ and $b_{1}^{q}-\sum_{i=2}^{n} b_{i}^{q}>0$.

One application of Aczél's inequality is the following Bellman's inequality [13]

$$
\left(a_{1}^{p}-\sum_{i=2}^{n} a_{i}^{p}\right)^{\frac{1}{p}}+\left(b_{1}^{p}-\sum_{i=2}^{n} b_{i}^{p}\right)^{\frac{1}{p}} \leq\left(\left(a_{1}+b_{1}\right)^{p}-\sum_{i=2}^{n}\left(a_{i}+b_{i}\right)^{p}\right)^{\frac{1}{p}} .
$$

Here $n$ is a positive integer, and $p \geq 1, a_{i}, b_{i}(i=1,2, \ldots, n)$ are positive numbers such that $a_{1}^{p}-\sum_{i=2}^{n} a_{i}^{p}>0$ and $b_{1}^{p}-\sum_{i=2}^{n} b_{i}^{p}>0$.

(c) 2012 Zhou; licensee Springer. This is an Open Access article distributed under the terms of the Creative Commons Attribution License (http://creativecommons.org/licenses/by/2.0), which permits unrestricted use, distribution, and reproduction in any medium, provided the original work is properly cited. 
In this paper, inspired by the functional generalizations of the Cauchy-Schwarz inequality [14] and of the Hölder inequality [15,16], we will establish some functional generalizations of the Aczél inequality and of the Bellman inequality. Refinements of these inequalities will also be presented.

As we seen, the following theorem is very useful to give results related to power sums and Aczél's inequality.

Theorem 1.1 (see e.g. $[9,17])$. Let $n$ be a positive integer, and $x_{i}(i=1,2, \ldots, n)$ be positive numbers such that $x_{1}-\sum_{i=2}^{n} x_{i}>0$.If $f: \mathbb{R}^{+} \rightarrow \mathbb{R}$ is a function such that $f(x) / x$ is increasing on $\mathbb{R}^{+}$, then

$$
f\left(x_{1}-\sum_{i=2}^{n} x_{i}\right) \leq f\left(x_{1}\right)-\sum_{i=2}^{n} f\left(x_{i}\right) .
$$

The inequality is reversed if $f(x) / x$ is decreasing on $\mathbb{R}^{+}$. The inequalities are strict if $f$ $(x) / x$ is strictly increasing or decreasing on $\mathbb{R}^{+}$.

Several mean value theorems for the related power sums of (4) have been established in $[9,18-20]$. In this paper, we will also generalize two of them in the last section.

\section{Aczél and Bellman's inequalities}

In order to establish the functional generalization of Aczél's inequality, we need the following lemma.

Lemma 2.1 (power means inequality, see [21]). Let $n$ be a positive integer, $p>0$ and let $a_{i}>0(i=1,2, \ldots, n)$. Then

$$
\sum_{i=1}^{n} a_{i}^{p} \leq n^{1-\min \{p, 1\}}\left(\sum_{i=1}^{n} a_{i}\right)^{p}
$$

Theorem 2.1. Let $n, m$ be positive integers, and let $p_{j} \geq 1, x_{i j}(i=1,2, \ldots, n ; j=1,2$, ..., $m$ ) be positive numbers such that $x_{1 j}-\sum_{i=2}^{n} x_{i j}>0$ for $j=1,2, \ldots$, m. If $f_{j}: \mathbb{R}^{+} \rightarrow \mathbb{R}$

+ is a function such that $f_{j}(x) / x$ is increasing on $\mathbb{R}^{+}$. Then we have

$$
\begin{aligned}
\prod_{j=1}^{m} f_{j}\left(x_{1 j}-\sum_{i=2}^{n} x_{i j}\right) & \leq \prod_{j=1}^{m}\left[\left(f_{j}\left(x_{1 j}\right)\right)^{p_{j}}-\sum_{i=2}^{n}\left(f_{j}\left(x_{i j}\right)\right)^{p_{j}}\right]^{\frac{1}{p_{j}}} \\
& \leq C \prod_{j=1}^{m} f_{j}\left(x_{1 j}\right)-\sum_{i=2}^{n} \prod_{j=1}^{m} f_{j}\left(x_{i j}\right),
\end{aligned}
$$

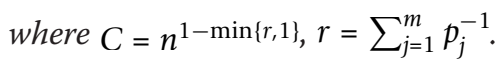

Proof. Applying Theorem 1.1 on each $f_{j}$ yields

$$
\prod_{j=1}^{m} f_{j}\left(x_{1 j}-\sum_{i=2}^{n} x_{i j}\right) \leq \prod_{j=1}^{m}\left(f_{j}\left(x_{1 j}\right)-\sum_{i=2}^{n} f_{j}\left(x_{i j}\right)\right) .
$$

Reusing Theorem 1.1 on $x^{p_{j}}$ and replacing $x_{i j}$ by $f_{j}\left(x_{i j}\right)$ in Theorem 1.1 , we obtain

$$
\prod_{j=1}^{m}\left(f_{j}\left(x_{1 j}\right)-\sum_{i=2}^{n} f_{j}\left(x_{i j}\right)\right) \leq \prod_{j=1}^{m}\left[\left(f_{j}\left(x_{1 j}\right)\right)^{p_{j}}-\sum_{i=2}^{n}\left(f_{j}\left(x_{i j}\right)\right)^{p_{j}}\right]^{\frac{1}{p_{j}}},
$$


which completes the first inequality of (6). To proof the second inequality of (6), let us denote

$$
\tilde{f}_{j}=\left[\left(f_{j}\left(x_{1 j}\right)\right)^{p_{j}}-\sum_{i=2}^{n}\left(f_{j}\left(x_{i j}\right)\right)^{p_{j}}\right]^{\frac{1}{p_{j}}} .
$$

By using the power means inequality (5) and the well known Hölder inequality, we have

$$
\begin{aligned}
\prod_{j=1}^{m} \tilde{f}_{j}+\sum_{i=2}^{n} \prod_{j=1}^{m} f_{j}\left(x_{i j}\right) & \leq n^{1-\min \{r, 1\}}\left(\prod_{j=1}^{m} \tilde{f}_{j}^{\frac{1}{r}}+\sum_{i=2}^{n} \prod_{j=1}^{m}\left(f_{j}\left(x_{i j}\right)\right)^{\frac{1}{r}}\right)^{r} \\
& \leq n^{1-\min \{r, 1\}}\left(\prod_{j=1}^{m}\left(\tilde{f}_{j}^{p_{j}}+\sum_{i=2}^{n}\left(f_{j}\left(x_{i j}\right)\right)\right)^{p_{j}} \frac{1}{r p_{j}}\right)^{r} \\
& =n^{1-\min \{r, 1\}} \prod_{j=1}^{m} f_{j}\left(x_{1 j}\right) .
\end{aligned}
$$

Rearranging the terms of (7) immediately leads to the second inequality of (6). This completes the proof. $\square$

Remark 2.1. From the proof we have that the second inequality of (6) still holds if $\left(f_{j}\left(x_{1 j}\right)\right)^{p_{j}}-\sum_{i=2}^{n}\left(f_{j}\left(x_{i j}\right)\right)^{p_{j}}>0$ for $p_{j}>0, j=1,2, \ldots, m$.

From Theorem 2.1, by taking $f_{j}(x)=x$, we get

Corollary 2.1. Under the assumptions of Theorem 2.1, and letting $f_{j}(x)=x$, we have

$$
\prod_{j=1}^{m}\left(x_{1 j}-\sum_{i=2}^{n} x_{i j}\right) \leq \prod_{j=1}^{m}\left(x_{1 j}^{p_{j}}-\sum_{i=2}^{n} x_{i j}^{p_{j}}\right)^{\frac{1}{p_{j}}} \leq C \prod_{j=1}^{m} x_{1 j}-\sum_{i=2}^{n} \prod_{j=1}^{m} x_{i j} .
$$

The first inequality of (8) gives a lower bound of Aczél's inequality. And the second is a generalized Aczél inequality obtained in [22].

The following theorem is the functional generalization of Bellman's inequality.

Theorem 2.2. Let $n, m$ be positive integers, and let $p \geq 1, x_{i j}(i=1,2, \ldots, n ; j=1,2$, ..., $m)$ be positive numbers such that $x_{1 j}-\sum_{i=2}^{n} x_{i j}>0$ for $j=1,2, \ldots$, m. If $f_{j}: \mathbb{R}^{+} \rightarrow \mathbb{R}^{+}$ is a function such that $f_{j}(x) / x$ is increasing on $\mathbb{R}^{+}$. Then we have

$$
\begin{aligned}
\sum_{j=1}^{m} f_{j}\left(x_{1 j}-\sum_{i=2}^{n} x_{i j}\right) & \leq \sum_{j=1}^{m}\left[\left(f_{j}\left(x_{1 j}\right)\right)^{p}-\sum_{i=2}^{n}\left(f_{j}\left(x_{i j}\right)\right)^{p}\right]^{\frac{1}{p}} \\
& \leq\left[\left(\sum_{j=1}^{m} f_{j}\left(x_{1 j}\right)\right)^{p}-\sum_{i=2}^{n}\left(\sum_{j=1}^{m} f_{j}\left(x_{i j}\right)\right)^{p}\right]^{\frac{1}{p}},
\end{aligned}
$$


Proof. The proof of the first inequality of (9) is similar to the proof of Theorem 2.1 and we omit it. The second inequality is an identity when $p=1$. Hence we only need to prove the second inequality of (9) for $p>1$ below.

From the assumptions and Theorem 1.1, we have

$$
f_{j}\left(x_{1 j}\right)-\sum_{i=2}^{n} f_{j}\left(x_{i j}\right)>f_{j}\left(x_{1 j}-\sum_{i=2}^{n} x_{i j}\right)>0, \quad j=1,2, \ldots, m .
$$

Applying the above inequality, the power means inequality (5) and the Minkowski inequality (see [21]), we obtain

$$
\left(\sum_{j=1}^{m} f_{j}\left(x_{1 j}\right)\right)^{p} \geq\left(\sum_{j=1}^{m}\left(\sum_{i=2}^{n}\left(f_{j}\left(x_{i j}\right)\right)^{p}\right)^{\frac{1}{p}}\right)^{p} \geq \sum_{i=2}^{n}\left(\sum_{j=1}^{m} f_{j}\left(x_{i j}\right)\right)^{p} .
$$

we now deduce from Theorem 2.1 that

$$
\begin{aligned}
& \left(\left(f_{l}\left(x_{1 l}\right)\right)^{p}-\sum_{i=2}^{n}\left(f_{l}\left(x_{i l}\right)\right)^{p}\right)^{\frac{1}{p}}\left[\left(\sum_{j=1}^{m} f_{j}\left(x_{1 j}\right)\right)^{p}-\sum_{i=2}^{n}\left(\sum_{j=1}^{m} f_{j}\left(x_{i j}\right)\right)^{p}\right]^{1-\frac{1}{p}} \\
& \leq f_{l}\left(x_{1 l}\right)\left(\sum_{j=1}^{m} f_{j}\left(x_{1 j}\right)\right)^{p-1}-\sum_{i=2}^{n} f_{l}\left(x_{i l}\right)\left(\sum_{j=1}^{m} f_{j}\left(x_{i j}\right)\right)^{\prime}
\end{aligned}
$$

for $l=1,2, \ldots, m$. This leads to

$$
\begin{aligned}
& \sum_{l=1}^{m}\left[\left(\left(f_{l}\left(x_{1 l}\right)\right)^{p}-\sum_{i=2}^{n}\left(f_{l}\left(x_{i l}\right)\right)^{p}\right)^{\frac{1}{p}}\right]\left[\left(\sum_{j=1}^{m} f_{j}\left(x_{1 j}\right)\right)^{p}-\sum_{i=2}^{n}\left(\sum_{j=1}^{m} f_{j}\left(x_{i j}\right)\right)^{p}\right]^{1-\frac{1}{p}} \\
& \leq \sum_{l=1}^{m} f_{l}\left(x_{1 l}\right)\left(\sum_{j=1}^{m} f_{j}\left(x_{1 j}\right)\right)^{p-1}-\sum_{i=2}^{n}\left(\sum_{l=1}^{m} f_{l}\left(x_{i l}\right)\right)\left(\sum_{j=1}^{m} f_{j}\left(x_{i j}\right)\right)^{p-1} \\
& =\left(\sum_{j=1}^{m} f_{j}\left(x_{1 j}\right)\right)^{p}-\sum_{i=2}^{n}\left(\sum_{j=1}^{m} f_{j}\left(x_{i j}\right)\right)^{p}
\end{aligned}
$$

which yields immediately the desired inequality. This completes the proof. $\square$ Taking $f_{j}(x)=x$ in Theorem 2.2, we obtain

Corollary 2.2. Under the assumptions of Theorem 2.2, and letting $f_{j}(x)=x$, we have

$$
\sum_{j=1}^{m}\left(x_{1 j}-\sum_{i=2}^{n} x_{i j}\right) \leq \sum_{j=1}^{m}\left(x_{1 j}^{p}-\sum_{i=2}^{n} x_{i j}^{p}\right)^{\frac{1}{p}} \leq\left[\left(\sum_{j=1}^{m} x_{1 j}\right)^{p}-\sum_{i=2}^{n}\left(\sum_{j=1}^{m} x_{i j}\right)^{p}\right]^{\frac{1}{p}} .
$$

The first inequality of (10) gives a lower bound of Bellman's inequality. And the second is a generalized Bellman inequality obtained in $[3,8]$.

Following the similar methods from $[8,10]$, we will establish some refinements of inequalities (6) and (9). Since the proofs are trivial by breaking the corresponding sums in the following form 


$$
x_{1 j}-\sum_{i=2}^{n} x_{i j}=x_{1 j}-\sum_{i=2}^{l} x_{i j}-\sum_{i=l+1}^{n} x_{i j},
$$

and reusing the corresponding theorems, we present these refinements below without proofs.

Theorem 2.3. Under the assumptions of Theorem 2.1, for $2 \leq l<n$, we have

$$
\begin{aligned}
\prod_{j=1}^{m}\left[f_{j}\left(x_{1 j}-\sum_{i=2}^{n} x_{i j}\right)\right] & \leq \prod_{j=1}^{m}\left[\left(f_{j}\left(x_{1 j}-\sum_{i=2}^{l} x_{i j}\right)\right)^{p_{j}}-\sum_{i=l+1}^{n}\left(f_{j}\left(x_{i j}\right)\right)^{p_{j}}\right]^{\frac{1}{p_{j}}} \\
& \leq \prod_{j=1}^{m}\left[\left(f_{j}\left(x_{1 j}\right)\right)^{p_{j}}-\sum_{i=2}^{n}\left(f_{j}\left(x_{i j}\right)\right)^{p_{j}}\right]^{\frac{1}{p_{j}}} \\
& \leq C_{1} \prod_{j=1}^{m}\left(\left(f_{j}\left(x_{1 j}\right)\right)^{p_{j}}-\sum_{i=2}^{l}\left(f_{j}\left(x_{i j}\right)\right)^{p_{j}}\right)^{\frac{1}{p_{j}}}-\sum_{i=l+1}^{n} \prod_{j=1}^{m} f_{j}\left(x_{i j}\right) \\
& \leq C_{1} C_{2} \prod_{j=1}^{m} f_{j}\left(x_{1 j}\right)-\sum_{i=2}^{n} \prod_{j=1}^{m} f_{j}\left(x_{i j}\right),
\end{aligned}
$$

where $C_{1}=(n-l+1)^{1-\min \{r, 1\}}, C_{2}=l^{1-\min \{r, 1\}}$. In particular $r=\sum_{j=1}^{m} p_{j}^{-1} \geq 1$, we have $C_{1}=C_{2}=1$, hence

$$
\begin{aligned}
\prod_{j=1}^{m}\left[\left(f_{j}\left(x_{1 j}\right)\right)^{p_{j}}-\sum_{i=2}^{n}\left(f_{j}\left(x_{i j}\right)\right)^{p_{j}}\right]^{\frac{1}{p_{j}}} & \leq \prod_{j=1}^{m}\left(\left(f_{j}\left(x_{1 j}\right)\right)^{p_{j}}-\sum_{i=2}^{l}\left(f_{j}\left(x_{i j}\right)\right)^{p_{j}}\right)^{\frac{1}{p_{j}}}-\sum_{i=l+1}^{n} \prod_{j=1}^{m} f_{j}\left(x_{i j}\right) \\
& \leq \prod_{j=1}^{m} f_{j}\left(x_{1 j}\right)-\sum_{i=2}^{n} \prod_{j=1}^{m} f_{j}\left(x_{i j}\right),
\end{aligned}
$$

leading to a refinement of (6).

Remark 2.2. The third and fourth inequality of (11) still holds if $\left(f_{j}\left(x_{1 j}\right)\right)^{p_{j}}-\sum_{i=2}^{n}\left(f_{j}\left(x_{i j}\right)\right)^{p_{j}}>0$ for $p_{j}>0, j=1,2, \ldots, m$.

Taking $f_{j}(x)=x$ in Theorem 2.3, we get

Corollary 2.3. Under the assumptions of Theorem 2.1 and letting $f_{j}(x)=x$, for $2 \leq l$ $<n$, we have

$$
\begin{aligned}
\prod_{j=1}^{m}\left(x_{1 j}-\sum_{i=2}^{n} x_{i j}\right) & \leq \prod_{j=1}^{m}\left[\left(x_{1 j}-\sum_{i=2}^{l} x_{i j}\right)^{p_{j}}-\sum_{i=l+1}^{n} x_{i j}^{p_{j}}\right]^{\frac{1}{p_{j}}} \\
& \leq \prod_{j=1}^{m}\left(x_{1 j}^{p_{j}}-\sum_{i=2}^{n} x_{i j}^{p_{j}}\right)^{\frac{1}{p_{j}}} \\
& \leq C_{1} \prod_{j=1}^{m}\left(x_{1 j}^{p_{j}}-\sum_{i=2}^{l} x_{i j}^{p_{j}}\right)^{\frac{1}{p_{j}}}-\sum_{i=l+1}^{n} \prod_{j=1}^{m} x_{i j} \\
& \leq C_{1} C_{2} \prod_{j=1}^{m} x_{1 j}-\sum_{i=2}^{n} \prod_{j=1}^{m} x_{i j} .
\end{aligned}
$$


In particular $r=\sum_{j=1}^{m} p_{j}^{-1} \geq 1$, we have

$$
\prod_{j=1}^{m}\left(x_{1 j}^{p_{j}}-\sum_{i=2}^{n} x_{i j}^{p_{j}}\right)^{\frac{1}{p_{j}}} \leq \prod_{j=1}^{m}\left(x_{1 j}^{p_{j}}-\sum_{i=2}^{l} x_{i j}^{p_{j}}\right)^{\frac{1}{p_{j}}}-\sum_{i=l+1}^{n} \prod_{j=1}^{m} x_{i j} \leq \prod_{j=1}^{m} x_{1 j}-\sum_{i=2}^{n} \prod_{j=1}^{m} x_{i j},
$$

leading (12) to a refinement of (8).

Remark 2.3. The third and fourth inequality of (12) still holds for $p_{j}>0$ if $x_{1 j}^{p_{j}}-\sum_{i=2}^{n} x_{i j}^{p_{j}}>0, j=1,2, \ldots, m$. The inequality (13) is also obtained in [8].

Theorem 2.4. Under the assumptions of Theorem 2.2, for $2 \leq l<n$, we have

$$
\begin{aligned}
\sum_{j=1}^{m} f_{j}\left(x_{1 j}-\sum_{i=2}^{n} x_{i j}\right) & \leq \sum_{j=1}^{m}\left[\left(f_{j}\left(x_{1 j}-\sum_{i=2}^{l} x_{i j}\right)\right)^{p}-\sum_{i=l+1}^{n}\left(f_{j}\left(x_{i j}\right)\right)^{p}\right]^{\frac{1}{p}} \\
& \leq \sum_{j=1}^{m}\left[\left(f_{j}\left(x_{1 j}\right)\right)^{p}-\sum_{i=2}^{n}\left(f_{j}\left(x_{i j}\right)\right)^{p}\right]^{\frac{1}{p}} \\
& \leq\left[\left(\sum_{j=1}^{m}\left(\left(f_{j}\left(x_{1 j}\right)\right)^{p}-\sum_{i=2}^{l}\left(f_{j}\left(x_{i j}\right)\right)^{p}\right)^{\frac{1}{p}}\right)^{p}-\sum_{i=l+1}^{n}\left(\sum_{j=1}^{m} f_{j}\left(x_{i j}\right)\right)^{p}\right]^{\frac{1}{p}} \\
& \leq\left[\left(\sum_{j=1}^{m} f_{j}\left(x_{1 j}\right)\right)^{p}-\sum_{i=2}^{n}\left(\sum_{j=1}^{m} f_{j}\left(x_{i j}\right)\right)^{p}\right]^{\frac{1}{p}}
\end{aligned}
$$

Taking $f_{j}(x)=x$ in Theorem 2.4, we have the following.

Corollary 2.4. Under the assumptions of Theorem 2.2, and letting $f_{j}(x)=x$, we have

$$
\begin{aligned}
\sum_{j=1}^{m}\left(x_{1 j}-\sum_{i=2}^{n} x_{i j}\right) & \leq \sum_{j=1}^{m}\left[\left(x_{1 j}-\sum_{i=2}^{l} x_{i j}\right)^{p}-\sum_{i=l+1}^{n} x_{i j}^{p}\right]^{\frac{1}{p}} \\
& \leq \sum_{j=1}^{m}\left(x_{1 j}^{p}-\sum_{i=2}^{n} x_{i j}^{p}\right)^{\frac{1}{p}} \\
& \leq\left[\left(\sum_{j=1}^{m}\left(x_{1 j}^{p}-\sum_{i=2}^{l} x_{i j}^{p}\right)^{\frac{1}{p}}\right)^{p}-\sum_{i=l+1}^{n}\left(\sum_{j=1}^{m} x_{i j}\right)^{p}\right]^{\frac{1}{p}} \\
& \leq\left[\left(\sum_{j=1}^{m} x_{1 j}\right)^{p}-\sum_{i=2}^{n}\left(\sum_{j=1}^{m} x_{i j}\right)^{p}\right]^{\frac{1}{p}} .
\end{aligned}
$$

The third and fourth inequality (15) is also obtained in [8]. 


\section{Mean value theorem}

As we seen, Theorem 1.1 is very useful to give results related to Aczél's inequality. It is also useful to give results related to power sums $[9,17]$. In this section, we first present a generalized version of Theorem 1.1, then use it to establish our first generalized mean value theorem related to power sums. We conclude this section with a mean value theorem which generalize the recent result obtained by Pečarić and Rehman [18].

Lemma 3.1. Let $n$ be a positive integer, and $x_{i}(i=1,2, \ldots, n)$ be positive numbers such that $x_{1}-\sum_{i=2}^{n} x_{i}>0$. If $f: \mathbb{R}^{+} \rightarrow \mathbb{R}$ is a function such that $f(x) / x^{p}$ is increasing on $\mathbb{R}^{+}$. Then the inequality (4) holds for $p \geq 1, f \geq 0$ on $\mathbb{R}^{+}$or $p \leq 1, f \leq 0$ on $\mathbb{R}^{+}$. If $f(x) / x^{p}$ is decreasing on $\mathbb{R}^{+}$, the inequality (4) is reversed for $p \geq 1, f \leq 0$ on $\mathbb{R}^{+}$or $p \leq 1, f \geq 0$ on $\mathbb{R}^{+}$.

Remark 3.1. If $p \neq 1, f \neq 0$ or $f(x) / x$ is strictly increasing or decreasing on $\mathbb{R}^{+}$, then strictly inequalities hold.

Proof. This Lemma is an easy corollary of Theorem 1.1, so we omit the proof. $\square$

Theorem 3.1. Let $p \geq 2$. Let $\left(x_{1}, x_{2}, \ldots, x_{n}\right) \in I^{n}$, where $I=[a, b] \subseteq(0, \infty)$ and $x_{1}-\sum_{i=2}^{n} x_{i} \in$ I. If $f: \mathbb{R}^{+} \rightarrow \mathbb{R}$ is a function such that $f \in C^{1}(I)$ and $m a^{p} \leq f(a) \leq M a^{p}$, where $m, M$ are defined by (17) below. Then there exists $\xi \in I$ such that

$$
f\left(x_{1}\right)-\sum_{i=2}^{n} f\left(x_{i}\right)-f\left(x_{1}-\sum_{i=2}^{n} x_{i}\right)=\frac{\xi f^{\prime}(\xi)-(p-1) f(\xi)}{\xi^{p}}\left[x_{1}^{p}-\sum_{i=2}^{n} x_{i}^{p}-\left(x_{1}-\sum_{i=2}^{n} x_{i}\right)^{p}\right] .
$$

Proof. Let

$$
F(x)=\frac{x f^{\prime}(x)-(p-1) f(x)}{x^{p}} .
$$

Since $I$ is compact and $f \in C^{1}(I)$, there exist $\tilde{x}, \hat{x} \in I$ such that

$$
M:=F(\tilde{x})=\max _{x \in I} F(x), \quad m:=F(\hat{x})=\min _{x \in I} F(x) .
$$

We define two auxiliary functions as follows

$$
\phi_{1}(x)=M x^{p}-f(x), \quad \phi_{2}(x)=f(x)-m x^{p} .
$$

It is easily deduced that

$$
\left(\frac{\phi_{1}(x)}{x^{p-1}}\right)^{\prime} \geq 0, \quad\left(\frac{\phi_{2}(x)}{x^{p-1}}\right)^{\prime} \geq 0,
$$

hence the two functions $\frac{\phi_{1}(x)}{x^{p-1}}$ and $\frac{\phi_{2}(x)}{x^{p-1}}$ are all increasing on $I$. From the above inequalities, we also have

$$
\phi_{1}^{\prime}(x)=\left(x^{p-1} \frac{\phi_{1}(x)}{x^{p-1}}\right)^{\prime}=\frac{p-1}{x} \phi_{1}(x)+x^{p-1}\left(\frac{\phi_{1}(x)}{x^{p-1}}\right)^{\prime} \geq \frac{p-1}{x} \phi_{1}(x),
$$

and

$$
\phi_{2}^{\prime}(x) \geq \frac{p-1}{x} \phi_{2}(x)
$$

By the famous Grownwall inequality and $\varphi_{1}(a) \geq 0$ and $\varphi_{2}(a) \geq 0$ from the assumption, we find 


$$
\phi_{1}(x) \geq 0, \quad \phi_{2}(x) \geq 0 .
$$

Now applying Lemma 3.1 on $\varphi_{1}(x)$ and $\varphi_{2}(x)$ respectively and rearranging the terms, we have

$$
\begin{aligned}
& f\left(x_{1}\right)-\sum_{i=2}^{n} f\left(x_{i}\right)-f\left(x_{1}-\sum_{i=2}^{n} x_{i}\right) \leq M\left[x_{1}^{p}-\sum_{i=2}^{n} x_{i}^{p}-\left(x_{1}-\sum_{i=2}^{n} x_{i}\right)^{p}\right], \\
& f\left(x_{1}\right)-\sum_{i=2}^{n} f\left(x_{i}\right)-f\left(x_{1}-\sum_{i=2}^{n} x_{i}\right) \geq m\left[x_{1}^{p}-\sum_{i=2}^{n} x_{i}^{p}-\left(x_{1}-\sum_{i=2}^{n} x_{i}\right)^{p}\right] .
\end{aligned}
$$

Applying Lemma 1.1 on the function $x^{p}$ we obtain

$$
x_{1}^{p}-\sum_{i=2}^{n} x_{i}^{p}-\left(x_{1}-\sum_{i=2}^{n} x_{i}\right)^{p}>0 .
$$

Combining (18) and (19) leads to

$$
m \leq \frac{f\left(x_{1}\right)-\sum_{i=2}^{n} f\left(x_{i}\right)-f\left(x_{1}-\sum_{i=2}^{n} x_{i}\right)}{x_{1}^{p}-\sum_{i=2}^{n} x_{i}^{p}-\left(x_{1}-\sum_{i=2}^{n} x_{i}\right)^{p}} \leq M .
$$

For our definition, $F(x)$ is continuous on $I$ and $m \leq F(x) \leq M$. Hence, there exists $\xi \in$ $I$ such that

$$
\frac{f\left(x_{1}\right)-\sum_{i=2}^{n} f\left(x_{i}\right)-f\left(x_{1}-\sum_{i=2}^{n} x_{i}\right)}{x_{1}^{p}-\sum_{i=2}^{n} x_{i}^{p}-\left(x_{1}-\sum_{i=2}^{n} x_{i}\right)^{p}}=\frac{\xi f^{\prime}(\xi)-(p-1) f(\xi)}{\xi^{p}},
$$

which immediately leads to (16). This completes the proof.

We present the Cauchy type mean value theorem of Theorem 3.1 below without proof for the proof is quite standard and coincides with the proof of Theorem 3.14 in [9].

Theorem 3.2. Let $p \geq 2$. Let $\left(x_{1}, x_{2}, \ldots, x_{n}\right) \in I^{n}$, where $I=[a, b] \subseteq(0, \infty)$ and $x_{1}-\sum_{i=2}^{n} x_{i} \in I$. If $f, g: \mathbb{R}^{+} \rightarrow \mathbb{R}$ are functions such that $f, g \in C^{1}(I)$ and $m_{f} a^{p} \leq f(a) \leq$ $M_{f} a^{p}, m_{g} a^{p} \leq g(a) \leq M_{g} a^{p}$, where $m_{f}, M_{f}$ and $m_{g}, M_{g}$ are defined by (17) with corresponding function $f$ and $g$. Then there exists $\xi \in I$ such that

$$
\begin{aligned}
& {\left[f\left(x_{1}\right)-\sum_{i=2}^{n} f\left(x_{i}\right)-f\left(x_{1}-\sum_{i=2}^{n} x_{i}\right)\right]\left[\xi g^{\prime}(\xi)-(p-1) g(\xi)\right]} \\
& \quad=\left[g\left(x_{1}\right)-\sum_{i=2}^{n} g\left(x_{i}\right)-g\left(x_{1}-\sum_{i=2}^{n} x_{i}\right)\right]\left[\xi f^{\prime}(\xi)-(p-1) f(\xi)\right] .
\end{aligned}
$$

Remark 3.2. If $p=2$, the conditions $m_{f} \leq \frac{f(a)}{a^{p}} \leq M_{f}, m_{g} \leq \frac{g(a)}{a^{p}} \leq M_{g}$ could be removed, then Theorem 3.1 and Theorem 3.2 reduce to Theorem 3.13 and Theorem 3.14 of [9], respectively.

We conclude this section with a generalization of the mean value theorem obtained in [18], which is a special case of the following theorem with $k=1$. As given in [18], this theorem is also a generalization of Theorem 3.1 with $p=2$.

Theorem 3.3. Let $\left(x_{1}, x_{2}, \ldots, x_{n}\right) \in I^{n}$, where $I$ is a compact interval, $p_{i}, q_{i}(i=1,2, \ldots$, $n)$ be non-negative numbers such that $\sum_{i=1}^{n} p_{i} x_{i} \in \operatorname{Iand} \sum_{i=1}^{n} p_{i} x_{i} \geq x_{j}, j=1,2$,..., n. If $f$ 
$L C^{k}(I)$, then there exists $\xi \in I$ such that

$$
\begin{aligned}
& \sum_{j=1}^{n} q_{j} f\left(\sum_{i=1}^{n} p_{i} x_{i}\right)-\sum_{j=1}^{n} q_{j} f\left(x_{j}\right) \\
& =\sum_{v=1}^{k-1} \sum_{j=1}^{n} q_{j} \frac{f^{(v)}\left(x_{j}\right)}{v !}\left(\sum_{i=1}^{n} p_{i} x_{i}-x_{j}\right)^{v}+\frac{f^{(k)}(\xi)}{k !} \sum_{j=1}^{n} q_{j}\left(\sum_{i=1}^{n} p_{i} x_{i}-x_{j}\right)^{k} .
\end{aligned}
$$

Proof. Since $I$ is compact and $f L C^{k}(I)$, there exist $\tilde{x}, \hat{x} \in I$ such that

$$
M:=f^{(k)}(\tilde{x})=\max _{x \in I} f^{(k)}(x), \quad m:=f^{(k)}(\hat{x})=\min _{x \in I} f^{(k)}(x) .
$$

We define $2 n$ auxiliary functions as follows

$$
\phi_{j}(x)=\frac{M}{k !}\left(x-x_{j}\right)^{k}+\sum_{\nu=0}^{k-1} \frac{f^{(v)}\left(x_{j}\right)}{v !}\left(x-x_{j}\right)^{\nu}-f(x),
$$

and

$$
\psi_{j}(x)=f(x)-\sum_{\nu=0}^{k-1} \frac{f^{(v)}\left(x_{j}\right)}{\nu !}\left(x-x_{j}\right)^{v}-\frac{m}{k !}\left(x-x_{j}\right)^{k},
$$

for $j=1,2, \ldots, n$. Then we have

$$
\phi_{j}^{\prime}(x)=\frac{M}{(k-1) !}\left(x-x_{j}\right)^{k-1}+\sum_{v=1}^{k-1} \frac{f^{(v)}\left(x_{j}\right)}{(v-1) !}\left(x-x_{j}\right)^{v-1}-f^{\prime}(x) .
$$

Expanding $f^{\prime}(x)$ at $x_{j}$ by the Taylor theorem, (25) can be rewritten as

$$
\phi_{j}^{\prime}(x)=\frac{M-f^{(k)}(\eta)}{(k-1) !}\left(x-x_{j}\right)^{k-1},
$$

where $\eta \in I$. Obviously, $\phi_{j}^{\prime}(x) \geq 0$ for $x \geq x_{j}, j=1,2, \ldots, n$, which means $\varphi_{j}(x)$ is increasing on $x \geq x_{j}$. Similarly, we can deduce that $\psi_{j}(x)$ is increasing on $x \geq x_{j}, j=1$, $2, \ldots, n$. Thus, from the assumption we obtain

$$
\phi_{j}\left(\sum_{i=1}^{n} p_{i} x_{i}\right) \geq \phi_{j}\left(x_{j}\right), \quad \psi_{j}\left(\sum_{i=1}^{n} p_{i} x_{i}\right) \geq \psi_{j}\left(x_{j}\right), \quad j=1,2, \ldots, n .
$$

Rearranging the terms yield

$$
f\left(\sum_{i=1}^{n} p_{i} x_{i}\right)-f\left(x_{j}\right)-\sum_{\nu=0}^{k-1} \frac{f^{(v)}\left(x_{j}\right)}{\nu !}\left(\sum_{i=1}^{n} p_{i} x_{i}-x_{j}\right)^{\nu} \leq \frac{M}{k !}\left(\sum_{i=1}^{n} p_{i} x_{i}-x_{j}\right)^{k},
$$

and

$$
f\left(\sum_{i=1}^{n} p_{i} x_{i}\right)-f\left(x_{j}\right)-\sum_{\nu=0}^{k-1} \frac{f^{(v)}\left(x_{j}\right)}{\nu !}\left(\sum_{i=1}^{n} p_{i} x_{i}-x_{j}\right)^{\nu} \geq \frac{m}{k !}\left(\sum_{i=1}^{n} p_{i} x_{i}-x_{j}\right)^{k},
$$


for $j=1,2, \ldots, n$. Hence,

$$
\sum_{j=1}^{n} q_{j}\left[f\left(\sum_{i=1}^{n} p_{i} x_{i}\right)-f\left(x_{j}\right)-\sum_{\nu=0}^{k-1} \frac{f^{(v)}\left(x_{j}\right)}{v !}\left(\sum_{i=1}^{n} p_{i} x_{i}-x_{j}\right)^{\nu}\right] \leq \frac{M}{k !} \sum_{j=1}^{n} q_{j}\left(\sum_{i=1}^{n} p_{i} x_{i}-x_{j}\right)^{k},
$$

and

$$
\sum_{j=1}^{n} q_{j}\left[f\left(\sum_{i=1}^{n} p_{i} x_{i}\right)-f\left(x_{j}\right)-\sum_{v=0}^{k-1} \frac{f^{(v)}\left(x_{j}\right)}{v !}\left(\sum_{i=1}^{n} p_{i} x_{i}-x_{j}\right)^{v}\right] \geq \frac{m}{k !} \sum_{j=1}^{n} q_{j}\left(\sum_{i=1}^{n} p_{i} x_{i}-x_{j}\right)^{k} .
$$

For $f \in C^{k}(I)$ and

$$
\frac{m}{k !} \sum_{j=1}^{n} q_{j}\left(\sum_{i=1}^{n} p_{i} x_{i}-x_{j}\right)^{k} \leq \frac{f^{(k)}(x)}{k !} \sum_{j=1}^{n} q_{j}\left(\sum_{i=1}^{n} p_{i} x_{i}-x_{j}\right)^{k} \leq \frac{M}{k !} \sum_{j=1}^{n} q_{j}\left(\sum_{i=1}^{n} p_{i} x_{i}-x_{j}\right)^{k},
$$

combining (26) and (27) immediately leads to (23). This completes the proof. $\square$

Similarly, we present the Cauchy type mean value theorem of Theorem 3.3 below without proof. This theorem reduce to the Cauchy type mean value theorem of [18] with $k=1$.

Theorem 3.4. Let $\left(x_{1}, x_{2}, \ldots, x_{n}\right) \in I^{n}$, where $I$ is a compact interval, $p_{i}, q_{i}(i=1,2, \ldots$, $n)$ be non-negative numbers such that $\sum_{i=1}^{n} p_{i} x_{i} \in I$ and $\sum_{i=1}^{n} p_{i} x_{i} \geq x_{j}, j=1,2, \ldots, n$. If $f$, $g \in C^{k}(I)$, then there exists $\xi \in I$ such that

$$
\begin{aligned}
& {\left[\sum_{j=1}^{n} q_{j} f\left(\sum_{i=1}^{n} p_{i} x_{i}\right)-\sum_{j=1}^{n} q_{j} f\left(x_{j}\right)-\sum_{\nu=1}^{k-1} \sum_{j=1}^{n} q_{j} \frac{f^{(v)}\left(x_{j}\right)}{\nu !}\left(\sum_{i=1}^{n} p_{i} x_{i}-x_{j}\right)^{\nu}\right] g^{(k)}(\xi)} \\
& =\left[\sum_{j=1}^{n} q_{j} g\left(\sum_{i=1}^{n} p_{i} x_{i}\right)-\sum_{j=1}^{n} q_{j} g\left(x_{j}\right)-\sum_{\nu=1}^{k-1} \sum_{j=1}^{n} q_{j} \frac{g^{(\nu)}\left(x_{j}\right)}{\nu !}\left(\sum_{i=1}^{n} p_{i} x_{i}-x_{j}\right)^{\nu}\right] f^{(k)}(\xi) .
\end{aligned}
$$

\section{Competing interests}

The authors declare that they have no competing interests.

Received: 9 January 2012 Accepted: 8 June 2012 Published: 8 June 2012

\section{References}

1. Aczél, J: Some general methods in the theory of functional equations in one variable. New applications of functional equations (in Russian). Uspekhi Matematicheskikh Nauk. 11(3):3-68 (1956)

2. Yang, W: Refinements of generalized Aczél-Popoviciu's inequality and Bellman's inequality. Comput Math Appl. 59(11):3570-3577 (2010). doi:10.1016/j.camwa.2010.03.050

3. Wu, S, Debnath, L: A new generalization of Aczél's inequality and its applications to an improvement of Bellman's inequality. Appl Math Lett. 21(6):588-593 (2008). doi:10.1016/j.aml.2007.07.010

4. Wu, S: A unified generalization of Aczél, Popoviciu and Bellman's inequalities. Taiwanese J Math. 14(4):1635-1646 (2010)

5. Vong, SW: On a generalization of Aczél's inequality. Appl Math Lett. 24, 1301-1307 (2011). doi:10.1016/.aml.2011.02.020

6. Vasić, PM, Pečarić, JE: On the Jensen inequality for monotone functions. Analele Universitatii din Timisoara. Seria Matematica-Informatica. 17, 95-104 (1979)

7. Losonczi, L, Páles, Z: Inequalities for indefinite forms. J Math Anal Appl. 205, 148-156 (1997). doi:10.1006/jmaa.1996.5188

8. Hu, Z, Xu, A: Re nements of Aczél and Bellman's inequalities. Comput Math Appl. 59(9):3078-3083 (2010). doi:10.1016/j. camwa.2010.02.027

9. Farid, G, Pečarić, J, Rehman, AU: On refinements of Aczél, Popoviciu, Bellman's inequalities and related results. J Inequal Appl 2010 (2010). Article ID 579567

10. Díaz-Barrero, JL, Grau-Sánchez, M, Popescu, PG: Refinements of Aczél, Popoviciu and Bellman's inequalities. Comput Math Appl. 56(9):2356-2359 (2008). doi:10.1016/j.camwa.2008.05.013

11. Cho, YJ, Matić, M, Pečarić, J: Improvements of some inequalities of Aczél's type. J Math Anal Appl. 259, $226-240$ (2001). doi:10.1006/jmaa.2000.7423

12. Popoviciu, T: On an inequality. Gaz Mat Fiz A. 11(64):451-461 (1959)

13. Bellman, R: On an inequality concerning an indefinite form. Am Math Monthly. 63, 108-109 (1956). doi:10.2307/2306434

14. Masjed-Jamei, M: A functional generalization of the Cauchy-Schwarz inequality and some subclasses. Appl Math Lett. 22(9):1335-1339 (2009). doi:10.1016/j.aml.2009.03.001 
15. Yang, W: A functional generalization of diamond- $a$ integral Hölder's inequality on time scales. Appl Math Lett. 23, 1208-1212 (2010). doi:10.1016/.j.aml.2010.05.013

16. Qiang, H, Hu, Z: Generalizations of Hölder's and some related inequalities. Comput Math Appl. 61, 392-396 (2011). doi:10.1016/j.camwa.2010.11.015

17. Pečarić, JE, Proschan, F, Tong, YL: Convex functions, partial orderings, and statistical applications. In Mathematics in Science and Engineering, vol. 187,Academic Press, Boston (1992)

18. Pečarić, J, Rehman, AU: On exponential convexity for power sums and related results. J Math Inequal. 5(2):265-274 (2011)

19. Pečarić, J, Rehman, AU: On logarithmic convexity for power sums and related results. J Inequal Appl 2008 (2008). Article ID 389410

20. Pečarić, J, Rehman, AU: On logarithmic convexity for power sums and related results II. J Inequal Appl 2008 (2008). Article ID 305623

21. Mitrinović, DS, Vasić, PM: Analytic Inequalities. Springer, New York (1970)

22. Wu, S, Debnath, L: Generalizations of Aczél's inequality and Popoviciu's inequality. Indian J Pure Appl Math. 36(2):49-62 (2005)

doi:10.1186/1029-242X-2012-130

Cite this article as: Zhou: Some generalizations of Aczél, Bellman's inequalities and related power sums. Journal of Inequalities and Applications 2012 2012:130.

\section{Submit your manuscript to a SpringerOpen ${ }^{\circ}$} journal and benefit from:

- Convenient online submission

- Rigorous peer review

- Immediate publication on acceptance

- Open access: articles freely available online

- High visibility within the field

- Retaining the copyright to your article

Submit your next manuscript at $\gg$ springeropen.com 